\title{
RELATIVE DIHEDRAL GROUP ACTIONS ON RATIONAL ELLIPTIC SURFACES
}

\author{
Shinzo BANNAI \\ (Received 2 September 2011)
}

\begin{abstract}
In this paper we give an explicit method for constructing every rational elliptic surface that has a four-torsion section. We use this construction to study the moduli space of birational equivalence classes of Galois covers that arise from rational elliptic surfaces with a relative dihedral group action. As a conclusion we find that the moduli space for such Galois covers whose Galois groups are the dihedral group of order eight is a nodal rational curve.
\end{abstract}

\section{Introduction}

Elliptic surfaces have been studied by many mathematicians from various points of views. Especially, elliptic surfaces with $D_{2 n}$-action appear in work done by H. Tokunaga [14-17] in the context of branched Galois coverings. Let $X, Y$ be normal projective varieties defined over the field of complex numbers $\mathbb{C}$. Let $\pi: X \rightarrow Y$ be a finite surjective morphism. Such morphisms $\pi$ are called covers of $Y$ and we also say that $X$ is a covering of $Y$. Additionally $\pi$ is said to be Galois if the field extension $\mathbb{C}(X) / \mathbb{C}(Y)$ of the rational function fields that is induced by $\pi$ is a Galois extension. For a fixed finite group $G$, we say that $\pi: X \rightarrow Y$ is a $G$-cover if it is Galois and $\operatorname{Gal}(X / Y) \cong G$. In this situation there is a natural $G$-action on $X$ and $X / G \cong Y$. The close relationship of $G$-covers and the Galois theory of function fields makes it natural to ask questions about birationally invariant properties of $G$-covers and it is important to classify $G$-covers up to birational equivalence.

Definition 1.1. Let $G$ be a finite group. Let $\pi: X \rightarrow Y$ and $\pi^{\prime}: X^{\prime} \rightarrow Y^{\prime}$ be $G$-covers. Then $\pi$ and $\pi^{\prime}$ are birationally equivalent if there exists a $G$-equivariant birational map $\phi: X \rightarrow X^{\prime}$ and a birational map $\psi: Y \rightarrow Y^{\prime}$ that satisfies $\pi^{\prime} \circ \phi=\psi \circ \pi$.

When considering Galois covers there are two basic problems. One of them is the 'construction problem' where one asks if a $G$-cover $\pi: X \rightarrow Y$ exists for a given finite group $G$ over a given algebraic variety $Y$. The other is the 'moduli problem' where one aims to classify $G$-covers for a given finite group $G$. Let $D_{2 n}$ be the dihedral group of order $2 n$. In [17], Tokunaga studied the 'construction problem' and proved an existence theorem of $D_{2 n}$-covers over $\mathbb{P}^{2}$ that are branched along a quartic and a line. T. Yasumura studied the birational equivalence of the $D_{6}$-covers that appear in [17] from a 'bottom-up' point of view, namely in terms of the branch curves of $\pi$ in $\mathbb{P}^{2}$. His result can be interpreted as the birational equivalence of certain elliptic surfaces with $D_{6}$-action [18]. In this paper we take a 'top-down'

2010 Mathematics Subject Classification: Primary 14E07; Secondary 14J27.

Keywords: Galois covers; Cremona group; elliptic surfaces.

(C) 2013 Faculty of Mathematics, Kyushu University 
point of view and study the birational equivalence of $G$-covers $\pi: X \rightarrow Y$ by looking at the covering surface $X$, where $X$ is a rational elliptic surface and $G$ acts relatively with respect to the elliptic fibration on $X$ and $G \cong D_{2 n}(n \geq 4)$.

By the definition of birational equivalence of $G$-covers, the classification of equivalence classes of $G$-covers is equivalent to the classification of birational equivalence classes of $G$-surfaces (i.e. surfaces with $G$-action). The case of rational $G$-surfaces has been studied by many mathematicians. J. Blanc [1] classified rational $G$-surfaces where $G$ is a finite abelian group. I. V. Dolgachev and V. A. Iskovskikh [2] studied the general case and essentially completed the classification of birational equivalence classes of rational $G$-surfaces for finite $G$. Their classification is done in two steps. In the first step they utilize the $G$-equivariant version of Mori-theory for surfaces and determine the structure of minimal $G$-surfaces and classify them up to isomorphism. In the second step, they use the fact that any $G$-equivariant birational map can be factored into $G$-equivariant links and use the classification of $G$-equivariant links to determine which minimal $G$-surfaces are birationally equivalent. Although the classification is essentially finished, there are still some details to be resolved as stated in [2, Section 9]. Also, since the classification is based on the classification of minimal $G$-surfaces, it is not easy to see where non-minimal surfaces fit. The elliptic $D_{2 n}$-surfaces that we consider in this paper are not minimal and one of the objectives of this paper is to clarify their birational classification. The main results are the following theorems. See Section 2 for the notation used here.

THeOREM 1.1. Let $\mathcal{E}$ be a rational elliptic $D_{8}$-surface. Then $\mathcal{E}$ is birationally equivalent to one and only one of the following $D_{8}$-surfaces.

(1) A rational elliptic surface of type 58 with $D_{8}$-action of Type 1 . Two surfaces of this type are birationally equivalent if and only if they are isomorphic as elliptic surfaces.

$\mathbb{P}^{1} \times \mathbb{P}^{1}$ with $D_{8}$-action given by

$$
\begin{aligned}
& \sigma\left(\left[y_{0}, y_{1}\right],\left[z_{0}, z_{1}\right]\right)=\left(\left[z_{0}, z_{1}\right],\left[y_{0}, y_{1}\right]\right), \\
& \tau\left(\left[y_{0}, y_{1}\right],\left[z_{0}, z_{1}\right]\right)=\left(\left[z_{0}, z_{1}\right],\left[y_{1}, y_{0}\right]\right) .
\end{aligned}
$$

(3) $\mathbb{P}^{1} \times \mathbb{P}^{1}$ with $D_{8}$-action given by

$$
\begin{aligned}
& \sigma\left(\left[y_{0}, y_{1}\right],\left[z_{0}, z_{1}\right]\right)=\left(\left[z_{0}-\sqrt{-1} z_{1}, \sqrt{-1} z_{0}-z_{1}\right],\left[y_{0}-\sqrt{-1} y_{1}, \sqrt{-1} y_{0}-y_{1}\right]\right), \\
& \tau\left(\left[y_{0}, y_{1}\right],\left[z_{0}, z_{1}\right]\right)=\left(\left[z_{0}, z_{1}\right],\left[y_{1}, y_{0}\right]\right) .
\end{aligned}
$$

THEOREM 1.2. The algebraic variety that parametrizes the birational equivalence classes of rational elliptic $D_{8}$-surfaces is a nodal rational curve.

This paper is organized as follows. In Section 2, we review basic facts on rational elliptic surfaces and consider their relative automorphism groups. In Section 3 we give an explicit method for constructing rational elliptic surfaces that have a four-torsion section. This construction allows us to describe the elliptic surfaces in detail. In Section 4 we state facts about the Mordell-Weil lattices of elliptic surfaces which enables us to calculate the intersection data of sections. In Section 5, we use the construction from Section 3 to determine the $G$-minimal models of the elliptic surfaces and determine their birational equivalence classes and prove Theorems 1.1 and 1.2. 


\section{Dihedral group actions on elliptic surfaces}

In this section, we review the definitions and basic facts about elliptic surfaces and discuss the conjugacy classes of finite subgroups of the relative automorphism group that are isomorphic to dihedral groups. We suppose that all surfaces are defined over $\mathbb{C}$. The references for this section are [4], [5], [12] and [13].

Definition 2.1. Let $\mathcal{E}$ be a smooth projective surface and let $C$ be a smooth projective curve. We say that $S$ is an elliptic surface if there exists a surjective morphism

$$
f: \mathcal{E} \rightarrow C
$$

such that:

(1) general fibers of $f$ are smooth curves of genus 1 ;

(2) $f$ is relatively minimal;

(3) $f$ has a distinguished section $O: C \rightarrow \mathcal{E}$ called the zero-section;

(4) $f$ has at least one singular fiber.

Since we assume the existence of a section, the generic fiber $E$ of $f: \mathcal{E} \rightarrow C$ can be viewed as an elliptic curve over $\mathbb{C}(C)$ and there is a natural one-to-one correspondence between sections of $f: \mathcal{E} \rightarrow C$ and $\mathbb{C}(C)$ rational points $E(\mathbb{C}(C))$ of $E$.

Definition 2.2. The set of sections of $f: \mathcal{E} \rightarrow C$ endowed with a group structure induced by the group structure of $E$ is called the Mordell-Weil group of $\mathcal{E}$ and is denoted by $\operatorname{MW}(\mathcal{E})$.

Note that the group structure on $E$ and on $\operatorname{MW}(\mathcal{E})$ depends on the choice of the zerosection. We often identify sections with the image curve and abuse language by referring to the curves as sections. We denote elements of $\operatorname{MW}(S)$ by lower case alphabets $s, t$. We must not confuse the group operation of $\operatorname{MW}(S)$ and the group operation as divisors. We use the notation $[s]$ when we want to emphasize that we are considering the curve corresponding to $s$. Under this notation, $s+t$ is a sum in $\operatorname{MW}(S)$ and $[s]+[t]$ is a sum as divisors.

Definition 2.3. An automorphism $\phi$ of $\mathcal{E}$ is called a relative automorphism of $f: \mathcal{E} \rightarrow C$ if it preserves the fibration, i.e. $f \circ \phi=f$. We denote the group of relative automorphisms of $\mathcal{E}$ by $\operatorname{Aut}_{C}(\mathcal{E})$.

Definition 2.4. We say that an elliptic surface $\mathcal{E}$ has a relative $G$-action if $G \subset \operatorname{Aut}_{C}(\mathcal{E})$. We also say that $\mathcal{E}$ is an elliptic $G$-surface.

We define an equivalence relation on elliptic $G$-surfaces.

Definition 2.5. Let $f: \mathcal{E} \rightarrow C, f^{\prime}: \mathcal{E}^{\prime} \rightarrow C$ be elliptic $G$-surfaces over $C$. We say that $\mathcal{E}, \mathcal{E}^{\prime}$ are isomorphic as elliptic $G$-surfaces if and only if there exists a $G$-equivariant isomorphism $\phi: \mathcal{E} \rightarrow \mathcal{E}^{\prime}$ that preserves the fibration (i.e. $f=f^{\prime} \circ \phi$ ).

The following facts are well known.

Lemma 2.1. [12, Chapter 3, Example 4.7] Any morphism of elliptic curves can be expressed as a composition of a translation and an isogeny. 
Lemma 2.2. [13, Chapter 3, Proposition 3.9] Let $\mathcal{E}$ and $\mathcal{E}^{\prime}$ be elliptic surfaces over $C$. Then there is a natural bijection between the set of dominant rational maps $\mathcal{E} \rightarrow \mathcal{E}^{\prime}$ over $C$ and the set of non-constant maps $E \rightarrow E^{\prime}$.

Lemma 2.3. [13, Chapter 3, Corollary 8.4.1] Let $f: \mathcal{E} \rightarrow C$ be a minimal fibered surface over $C$, and let $\tau: \mathcal{E} \rightarrow \mathcal{E}$ be a birational map commuting with the map to $C$ (i.e. $f \circ \tau=f$ ). Then $\tau$ is a morphism.

Lemma 2.4. [13, Chapter 3, Proposition 9.1] For any point $P \in \operatorname{MW}(\mathcal{E})(=E(\mathbb{C}(C)))$, the translation-by-P map extends to a automorphism of $\mathcal{E}$. The map $\operatorname{MW}(\mathcal{E}) \rightarrow \operatorname{Aut}_{C}(\mathcal{E})$ is a homomorphism.

The homomorphism in Lemma 2.4 turns out to be injective, and we identify $\operatorname{MW}(\mathcal{E})$ with its image in $\operatorname{Aut}_{C}(\mathcal{E})$. Let $\operatorname{Aut}_{O}(E)$ denote the automorphism group of $E$ (as a group). In the following we assume that $\operatorname{Aut}_{O}(E)=\mathbb{Z} / 2 \mathbb{Z}$ and denote the involution by $\iota$.

Proposition 2.1. Let $f: \mathcal{E} \rightarrow C$ be an elliptic surface. Then $\operatorname{Aut}_{C}(\mathcal{E})$ is generated by $\mathrm{MW}(\mathcal{E})$ and $\iota$. Furthermore, $\iota \circ s=(-s) \circ \iota$ for any $s \in \mathrm{MW}(\mathcal{E})$.

LEMmA 2.5. Let $g \in \operatorname{Aut}_{C}(S)$. Then $g$ has finite order if and only if either:

(1) $g \in \mathrm{MW}(\mathcal{E})_{\text {tor }}$; or

(2) $g=\iota \circ$ for some $t \in \mathrm{MW}(\mathcal{E})$.

Proof. Any element of the form (1) or (2) has finite order. Conversely suppose $g$ has finite order. If $g \in \mathrm{MW}(\mathcal{E})$, then $g \in \mathrm{MW}(\mathcal{E})_{\text {tor }}$ by definition of $\mathrm{MW}(\mathcal{E})_{\text {tor }}$. If $g \notin \mathrm{MW}(\mathcal{E})$ then by Lemma 2.1, $g=\iota \circ t$ for some $t \in \operatorname{MW}(\mathcal{E})$.

LEMma 2.6. The automorphisms $\iota \circ t$ and $\iota \circ t^{\prime}$ are conjugate in $\operatorname{Aut}_{C}(\mathcal{E})$ if and only if $t-t^{\prime}$ is 2-divisible in $\mathrm{MW}(\mathcal{E})$.

Proof. Suppose there exists $s \in \mathrm{MW}(\mathcal{E})$ such that $2 s=t-t^{\prime}$. Then $(-s) \circ\left(\iota \circ t^{\prime}\right) \circ s=$ $\iota \circ\left(2 s+t^{\prime}\right)=\iota \circ t$. Hence $\iota \circ t$ and $\iota \circ t^{\prime}$ are conjugate in $\operatorname{Aut}_{C}(\mathcal{E})$.

Conversely if $\iota \circ t$ and $\iota \circ t^{\prime}$ are conjugate in $\operatorname{Aut}_{C}(\mathcal{E})$, there exists $g \in \operatorname{Aut}_{C}(\mathcal{E})$ such that $g^{-1}\left(\iota \circ t^{\prime}\right) g=\iota \circ t$. If $g=s$ then $g^{-1}\left(\iota \circ t^{\prime}\right) g=(-s) \circ\left(\iota \circ t^{\prime}\right) \circ s=\iota \circ\left(2 s+t^{\prime}\right)=\iota \circ t$. Hence $t-t^{\prime}=2 s$. If $g=\iota \circ s$, then $g^{-1}\left(\iota \circ t^{\prime}\right) g=(\iota \circ s) \circ\left(\iota \circ t^{\prime}\right) \circ(\iota \circ s)=\iota \circ\left(2 s-t^{\prime}\right)=$ $\iota \circ t$. Hence $t+t^{\prime}=2 s$. But then we have $t-t^{\prime}=t+t^{\prime}-2 t^{\prime}=2 s-2 t^{\prime}=2\left(s-t^{\prime}\right)$. Hence $t-t^{\prime}$ is 2-divisible in $\operatorname{MW}(\mathcal{E})$.

The following lemma can be proved in a similar way.

Lemma 2.7. Let $t, t^{\prime} \in \operatorname{MW}(\mathcal{E})$. Then $t, t^{\prime}$ are conjugate in $\operatorname{Aut}_{C}(\mathcal{E})$ if and only if $t=t^{\prime}$ or $t=-t^{\prime}$.

LEMMA 2.8. Let $t$ be an $n$-torsion element of $\mathrm{MW}(\mathcal{E})$ and let $s$ be any element of $\mathrm{MW}(\mathcal{E})$. Then the subgroup generated by $\iota \mathrm{s}$ and $t$ is isomorphic to a dihedral group of order $2 n$ :

$$
\langle\iota \circ s, t\rangle \cong D_{2 n} .
$$

Conversely, any subgroup of $\operatorname{Aut}_{C}(\mathcal{E})$ isomorphic to $D_{2 n}$ is generated by $1 \circ s, t$ for some $s, t \in \operatorname{MW}(\mathcal{E})$. 
Proof. We have relations $(\iota \circ s)^{2}=t^{n}=((\iota \circ s) \circ t)^{2}=1$, hence $G=\langle\iota \circ s, t\rangle$ is a quotient of $D_{2 n}$, but $G$ has order $2 n$ which implies $G=D_{2 n}$.

Since finite elements of $\operatorname{Aut}_{C}(\mathcal{E})$ are of the form $t \in \mathrm{MW}(\mathcal{E})$ or $\iota \circ s$ for some $s \in \mathrm{MW}(S)$, and since $D_{2 n} \not \subset \mathrm{MW}(\mathcal{E})$ since it is non-abelian, we see that any subgroup isomorphic to $D_{2 n}$ is generated by $\iota \circ s, t$ for some $s, t \in \mathrm{MW}(\mathcal{E})$.

The above lemmas show that it is important to know the structure of the MordellWeil group and especially the torsion part of the Mordell-Weil group in order to study the conjugacy classes of relative dihedral group actions on an elliptic surface. For rational elliptic surfaces, the structure has been calculated by U. Persson [9] and many other mathematicians and is well known. In Table 1 we list the surfaces having a torsion section of order $n$ for $n \geq 4$. The type of $S$ corresponds to the number in Oguiso and Shioda's list [8] which gives the structure of the Mordell-Weil lattice of $S$, which will be used later.

TABLE 1. Rational elliptic surfaces with torsion.

\begin{tabular}{cccc}
\hline & & $\begin{array}{c}\text { Number of isomorphism } \\
\text { classes of } \mathcal{E}\end{array}$ & Type of $\mathcal{E}$ \\
\hline 6 & $\mathrm{MW}(S)$ & 1 & 66 \\
5 & $\mathbb{Z} / 6 \mathbb{Z}$ & 1 & 67 \\
4 & $\mathbb{Z} / 5 \mathbb{Z}$ & 2 & 70,72 \\
& $\mathbb{Z} / 4 \mathbb{Z}$ & 1 & 74 \\
& $\mathbb{Z} \oplus \mathbb{Z} / 4 \mathbb{Z}$ & $\infty$ & 58 \\
\hline
\end{tabular}

From Table 1 and the above lemmas, we are able to classify rational elliptic $G$-surfaces up to isomorphism (as elliptic $G$-surfaces) for $G \cong D_{2 n}(n \geq 4)$. If $\operatorname{MW}(\mathcal{E}) \cong \mathbb{Z} / n \mathbb{Z}$ then Aut $_{C}(\mathcal{E}) \cong D_{2 n}$ and there is a unique relative $D_{2 n}$-action on the surface. For the other cases, we have the following lemmas.

PROPOSITION 2.2. Let $\mathcal{E}$ be the elliptic surface of type 74 such that

$$
\operatorname{MW}(\mathcal{E}) \cong \mathbb{Z} / 2 \mathbb{Z} \oplus \mathbb{Z} / 4 \mathbb{Z}
$$

Then there are four isomorphism classes of relative $D_{8}$-actions on $\mathcal{E}$.

Proof. Let $D_{8}=\langle\iota \circ s, t\rangle$ for $s, t \in \mathrm{MW}(\mathcal{E})$, where $t$ is an element of order 4. From Lemma 2.7, we see that there are two non-conjugate four-torsion elements $(0,1)$ and $(1,1)$ in $\mathbb{Z} / 2 \mathbb{Z} \oplus \mathbb{Z} / 4 \mathbb{Z}$. Then we can suppose that $s=(0,0)$ or $(1,0)$ by translating by the torsion element if necessary. Then $\iota \circ(0,0)=\iota$ and $\iota \circ(1,0)$ are not conjugate by Lemma 2.6. Hence we have four non-conjugate relative $D_{8}$-actions on $\mathcal{E}$, namely $\langle\iota, t\rangle,\left\langle\iota, t^{\prime}\right\rangle,\langle\iota \circ s, t\rangle,\left\langle\iota \circ s, t^{\prime}\right\rangle$, where $s=(1,0), t=(0,1), t^{\prime}=(1,1) \in \operatorname{MW}(\mathcal{E})$.

Note that even though $(\mathcal{E},\langle\iota, t\rangle)$ and $\left(\mathcal{E},\left\langle\iota, t^{\prime}\right\rangle\right)$ are not isomorphic as elliptic $D_{8}$ surfaces, they are isomorphic as $D_{8}$-surfaces. There exists an involution on the base that induces an $D_{8}$-equivariant isomorphism that does not preserve the fibration.

PROPOSITION 2.3. Let $\mathcal{E}$ be a rational elliptic surface with $\mathrm{MW}(\mathcal{E}) \cong \mathbb{Z} \oplus \mathbb{Z} / 4 \mathbb{Z}$. Then there are two isomorphism classes of relative $D_{8}$-actions on $\mathcal{E}$. 
Proof. As in Proposition 2.2 we can choose generators $\iota \circ s, t$ and suppose that $s=(m, 0)$ for some $m \in \mathbb{Z}$. Then $\iota \circ s$ is conjugate to $\iota$ or $\iota \circ(1,0)$ depending on the parity of $m$. Hence there are two isomorphism classes of relative $D_{8}$-actions on $S$ represented by $\langle\iota, t\rangle$ and $\langle\iota \circ s, t\rangle$ where $s=(1,0)$ and $t=(0,1)$.

Definition 2.6. Let $\mathcal{E}$ be a rational elliptic surface of type 58 or 74 . Then we call $D_{8}$-actions on $\mathcal{E}$ that are isomorphic to $\langle\iota, t\rangle$ for some four-torsion section $t$, actions of type 1 . We call $D_{8}$-actions that are not isomorphic to $\langle\iota, t\rangle$ actions of type 2 .

\section{Rational elliptic surfaces with four-torsion section}

In this section we give a method for explicitly constructing every rational elliptic surface that has a four-torsion section. We do this by blowing-up certain pencils of curves of genus 1 of $\mathbb{P}^{1} \times \mathbb{P}^{1}$. We use Kodaira's notation for singular fibers of elliptic surfaces.

Let $\left(\left[y_{0}, y_{1}\right],\left[z_{0}, z_{1}\right]\right)$ be homogeneous coordinates of $\mathbb{P}^{1} \times \mathbb{P}^{1}$. We define a $D_{8} \cong$ $\left\langle\sigma, \tau \mid \sigma^{2}=\tau^{4}=(\sigma \tau)^{2}=1\right\rangle$ action on $\mathbb{P}^{1} \times \mathbb{P}^{1}$ as

$$
\begin{aligned}
& \sigma\left(\left[y_{0}, y_{1}\right],\left[z_{0}, z_{1}\right]\right)=\left(\left[z_{0}, z_{1}\right],\left[y_{0}, y_{1}\right]\right), \\
& \tau\left(\left[y_{0}, y_{1}\right],\left[z_{0}, z_{1}\right]\right)=\left(\left[z_{0}, z_{1}\right],\left[y_{1}, y_{0}\right]\right) .
\end{aligned}
$$

Let $\pi: \mathbb{P}^{1} \times \mathbb{P}^{1} \rightarrow \mathbb{P}^{1} \times \mathbb{P}^{1} / D_{8}$ be the quotient morphism.

LEMMA 3.1. The quotient of $\mathbb{P}^{1} \times \mathbb{P}^{1}$ by the $D_{8}$-action given above is isomorphic to $\mathbb{P}^{2}$. Furthermore, $\pi$ can be factored into a bi-double cover $\pi_{2}: \mathbb{P}^{1} \times \mathbb{P}^{1} \rightarrow \mathbb{P}^{1} \times \mathbb{P}^{1}$ and a double cover $\pi_{1}: \mathbb{P}^{1} \times \mathbb{P}^{1} \rightarrow \mathbb{P}^{2}$.

Proof. Let $G$ be the subgroup of $D_{8}$ generated by $\sigma \tau$ and $\sigma \tau^{3}$. Then $G$ is a normal subgroup of $D_{8}, G \cong(\mathbb{Z} / 2 \mathbb{Z})^{2}$ and $D_{8} / G \cong \mathbb{Z} / 2 \mathbb{Z}$. Hence $\pi$ can be factored into a bi-double cover $\pi_{2}: \mathbb{P}^{1} \times \mathbb{P}^{1} \rightarrow \mathbb{P}^{1} \times \mathbb{P}^{1} / G$, and a double cover $\pi_{1}: \mathbb{P}^{1} \times \mathbb{P}^{1} / G \rightarrow \mathbb{P}^{1} \times \mathbb{P}^{1} / D_{8}$. Since the actions of $\sigma \tau$ and $\sigma \tau^{3}$ are given by

$$
\begin{aligned}
\sigma \tau\left(\left[y_{0}, y_{1}\right],\left[z_{0}, z_{1}\right]\right) & =\left(\left[y_{1}, y_{0}\right],\left[z_{0}, z_{1}\right]\right), \\
\sigma \tau^{3}\left(\left[y_{0}, y_{1}\right],\left[z_{0}, z_{1}\right]\right) & =\left(\left[y_{0}, y_{1}\right],\left[z_{1}, z_{0}\right]\right),
\end{aligned}
$$

it is immediate that $\mathbb{P}^{1} \times \mathbb{P}^{1} / G \cong \mathbb{P}^{1} \times \mathbb{P}^{1}$ and that $D_{8} / G$ acts on $\mathbb{P}^{1} \times \mathbb{P}^{1} / G \cong \mathbb{P}^{1} \times \mathbb{P}^{1}$ by interchanging the rulings. Hence $\mathbb{P}^{1} \times \mathbb{P}^{1} / D_{8} \cong\left(\mathbb{P}^{1} \times \mathbb{P}^{1} / G\right) /\left(D_{8} / G\right) \cong \mathbb{P}^{2}$.

The following corollaries follow immediately from Lemma 3.1.

COROLlary 3.1. $\pi$ is defined by a $D_{8}$-invariant linear system $\Lambda$ consisting of curves in $\mathbb{P}^{1} \times \mathbb{P}^{1}$ of bi-degree $(2,2)$.

COROLlARY 3.2. The branch divisor of $\pi$ consists of a non-singular conic $B$ and two lines $L_{1}, L_{2}$ each of which are tangent to $B$.

Let

$$
\begin{aligned}
& C_{0}: f_{0}=y_{0} y_{1} z_{0} z_{1}, \\
& C_{1}: f_{1}=y_{0}^{2} z_{0}^{2}+y_{0}^{2} z_{1}^{2}+y_{1}^{2} z_{0}^{2}+y_{1}^{2} z_{1}^{2}, \\
& C_{2}: f_{2}=y_{0} y_{1} z_{0}^{2}+y_{0} y_{1} z_{1}^{2}+y_{0}^{2} z_{0} z_{1}+y_{1}^{2} z_{0} z_{1} .
\end{aligned}
$$


Then $\left\{C_{0}, C_{1}, C_{2}\right\}$ is a basis for $\Lambda$. Let $\left[x_{0}, x_{1}, x_{2}\right]$ be homogeneous coordinates of $\mathbb{P}^{2}$. If we fix coordinates so that $\pi: \mathbb{P}^{1} \times \mathbb{P}^{1} \rightarrow \mathbb{P}^{2}$ is given by

$$
\pi\left(\left[y_{0}, y_{1}\right],\left[z_{0}, z_{1}\right]\right)=\left(f_{0}, f_{1}, f_{2}\right) \in \mathbb{P}^{2}
$$

the defining equations of $B, L_{1}$ and $L_{2}$ are given by

$$
\begin{aligned}
& B: 4 x_{0} x_{1}-x_{2}^{2}=0, \\
& L_{1}: 4 x_{0}+x_{1}+2 x_{2}=0, \\
& L_{2}: 4 x_{0}+x_{1}-2 x_{2}=0 .
\end{aligned}
$$

There is a one-to-one correspondence between members of $\Lambda$ and lines of $\mathbb{P}^{2}$. For a member $C \in \Lambda$, we denote the corresponding line by $l_{C}$. In general $C$ is a smooth curve of genus 1 . $C$ becomes singular, reducible, non-reduced depending on how $l_{C}$ intersects the branch divisor of $\pi$. Let $q_{0}=L_{1} \cap L_{2}$ and $q_{i}=B \cap L_{i}(i=1,2)$.

LEMMA 3.2. The structure of $C$ is given in Table 2. $I_{1}$ is a nodal rational curve. $I_{n}(n \geq 2)$ is a cycle of $n$ smooth rational curves. $h_{i}, v_{i}(i=0,1)$ are horizontal and vertical fibers of $\mathbb{P}^{1} \times \mathbb{P}^{1}$.

TABLE 2. The structure of $C$.

\begin{tabular}{cc}
\hline$l_{C}$ & $C=\pi^{*}\left(l_{C}\right)$ \\
\hline$q_{1} \in l_{C}, q_{0}, q_{2} \notin l$ & $I_{1}$ \\
$q_{2} \in l_{C}, q_{0}, q_{1} \notin l$ & $I_{1}$ \\
$q_{0} \in l_{C}, q_{1}, q_{2} \notin l$ & $I_{2}$ \\
$q_{1}, q_{2} \in l_{C}$ & $I_{2}$ \\
$q_{0}, q_{1} \in l_{C}$ & $2 h_{0}+2 v_{0}$ (non-reduced) \\
$q_{0}, q_{2} \in l_{C}$ & $2 h_{1}+2 v_{1}$ (non-reduced) \\
$q_{i} \notin l_{C}, l_{C}$ is tangent to $B$ & $I_{4}$ \\
$l_{C}$ : general & smooth of genus one \\
\hline
\end{tabular}

Since general members of $\Lambda$ are smooth curves of genus 1 , we can construct elliptic surfaces by blowing-up the base points of sub-pencils of $\Lambda$. Let $\lambda$ be a sub-pencil of $\Lambda$. Then $\lambda$ corresponds to a pencil of lines in $\mathbb{P}^{2}$. Let $q_{\lambda}$ be the base point of this pencil of lines. We can identify the dual line $\tilde{l}_{q_{\lambda}}$ of $q_{\lambda}$ with the base curve of the elliptic surface that is obtained by blowing-up the base points of $\lambda$.

LEMMA 3.3. The surface $\mathcal{E}_{\lambda}$ obtained by blowing-up the base points of a sub-pencil $\lambda$ of $\Lambda$ is a rational elliptic surface if and only if $q_{\lambda} \neq q_{0}, q_{1}, q_{2}$. Furthermore, the number of the base points of $\lambda$, the configuration of singular fibers and the Mordell-Weil group of $\mathcal{E}_{\lambda}$ is given by Table 3 .

Proof. If $q_{\lambda}=p_{i}$ for some $i$, then every curve in $\lambda$ becomes singular by Lemma 3.2. If $q_{\lambda} \neq q_{0}, q_{1}, q_{2}$, then there are only a finite number of singular members of $\lambda$ and general members of $\lambda$ are smooth curves of genus 1 . Hence $\mathcal{E}_{\lambda}$ is an elliptic surface if and only if $q_{\lambda} \neq q_{0}, q_{1}, q_{2}$. The configuration of singular fibers can be computed easily and is left to the reader. The Mordell-Weil group for each type of surfaces is given in [9]. 
TABLE 3. The structure of singular fibers.

\begin{tabular}{cccc}
\hline$q_{\lambda}$ & $\left|\pi^{-1}\left(q_{\lambda}\right)\right|$ & Singular fibers & $\mathrm{MW}\left(\mathcal{E}_{\lambda}\right)$ \\
\hline$q_{\lambda} \in B \backslash\left\{q_{1}, q_{2}\right\}$ & 4 & $I_{8}, I_{2}, I_{1}, I_{1}$ & $\mathbb{Z} / 4 \mathbb{Z}$ \\
$q_{\lambda} \in L_{i} \backslash\left\{q_{0}, q_{i}\right\}, i=1,2$ & 4 & $I_{1}^{*}, I_{4}, I_{1}$ & $\mathbb{Z} / 4 \mathbb{Z}$ \\
$q_{\lambda} \in \overline{q_{1} q_{2}} \backslash\left\{q_{1}, q_{2}\right\}$ & 8 & $I_{4}, I_{4}, I_{2}, I_{2}$ & $\mathbb{Z} / 4 \mathbb{Z} \oplus \mathbb{Z} / 2 \mathbb{Z}$ \\
$q_{\lambda}:$ general & 8 & $I_{4}, I_{4}, I_{2}, I_{1}, I_{1}$ & $\mathbb{Z} \oplus \mathbb{Z} / 4 \mathbb{Z}$ \\
\hline
\end{tabular}

Let $l_{C}$ be defined by $\alpha x_{0}+\beta x_{1}+\gamma x_{2}=0$. Then $C$ is the curve defined by $\alpha f_{0}+\beta f_{1}+$ $\gamma f_{2}=0$. The $j$-invariant $j_{C}$ of $C$ can be computed directly by using Maple, and is given by

$$
j_{C}=\frac{\left(\alpha^{4}-16 \alpha^{2} \beta^{2}-16 \alpha^{2} \gamma^{2}+96 \alpha \beta \gamma^{2}+256 \beta^{4}-256 \beta^{2} \gamma^{2}+16 \gamma^{4}\right)^{3}}{(\alpha-4 \beta)^{2}(\alpha+4 \beta+4 \gamma)(\alpha+4 \beta-4 \gamma)\left(\alpha \beta-\gamma^{2}\right)^{4}} .
$$

We can view this as a rational function on the dual projective space $\tilde{\mathbb{P}}^{2}$ with homogeneous coordinates $[\alpha, \beta, \gamma]$. The $j$-function of the elliptic surfaces in Lemma 3.3 can be obtained by restricting this rational function to the dual line $\tilde{l}_{q_{\lambda}}$ of $q_{\lambda}$ which can be identified with the base curve of $\mathcal{E}_{\lambda}$.

Let $X$ be the extremal rational elliptic surface of type 72 whose singular fibers are $I_{1}^{*}, I_{4}, I_{1}$. A detailed description of $X$ can be found in [7]. Let $[u, v]$ be homogeneous coordinates of the base curve $\mathbb{P}_{X}^{1}$ of $X$ and suppose that the $I_{1}^{*}, I_{4}, I_{1}$ lie over $[2,1],[-2,1],[1,0]$, respectively. Then the $j$-function of $X$ is given by

$$
j_{X}=\frac{256\left(u^{2}-3 v^{2}\right)^{3}}{v^{4}\left(u^{2}-4 v^{2}\right)} .
$$

If we consider a base change $\mathbb{P}^{1} \rightarrow \mathbb{P}_{X}^{1}$ of degree 2 branched over $[2,1]$ and another point $q \notin\{[-2,1],[1,0]\}$ then we obtain an elliptic surface of type 58. It is well known (see $[\mathbf{7}, \mathbf{1 0}]$ ) that any $\mathcal{E}$ with a four-torsion section can be obtained by such base change.

On the other hand, if we define $\psi: \tilde{\mathbb{P}}^{2} \rightarrow \mathbb{P}_{X}^{1}$ by

$$
[\alpha, \beta, \gamma] \rightarrow\left[\alpha^{2}+16 \beta^{2}-8 \gamma^{2},-4 \alpha \beta+4 \gamma^{2}\right],
$$

then $j_{C}=j_{X} \circ \psi$. Hence if we restrict $\psi$ to $\tilde{l}_{q_{\lambda}}$ we find the base change morphism $\left.\psi\right|_{\tilde{l}_{q_{\lambda}}}$ : $\tilde{l}_{q_{\lambda}} \rightarrow \mathbb{P}_{X}^{1}$ that gives $\mathcal{E}_{\lambda}$. Since

$$
\left(\alpha^{2}+16 \beta^{2}-8 \gamma^{2}\right)-2\left(-4 \alpha \beta+4 \gamma^{2}\right)=(\alpha-4 \beta)^{2},
$$

$\left.\psi\right|_{\tilde{q}_{\lambda}}$ is branched over $[2,1]$ for all $\lambda$. The other branch point is given by $\left[r_{0}, r_{1}\right]$, where $\tilde{l}_{q_{\lambda}}$ is tangent to

$$
C_{r}: r_{1}\left(\alpha^{2}+16 \beta^{2}-8 \gamma^{2}\right)-r_{0}\left(4 \alpha \beta-4 \gamma^{2}\right)=0 .
$$

Conversely for any $\left[r_{0}, r_{1}\right] \in \mathbb{P}_{X}$, we can find a line $\tilde{l}$ tangent to $C_{r}$ and $\left.\psi\right|_{\tilde{l}}$ is branched over $[2,1]$ and $\left[r_{0}, r_{1}\right]$. Hence every base change morphism that gives a surface of type 58 can be found and this implies that every surface of type 58 can be constructed by blowing-up a sub-pencil $\lambda$.

We can also see when we obtain isomorphic surfaces. For $\mathcal{E}_{\lambda}$ and $\mathcal{E}_{\lambda^{\prime}}$ of type 58, they are isomorphic if and only if $\tilde{l}_{q_{\lambda}}$ and $\tilde{l}_{q_{\lambda^{\prime}}}$ are both tangent to the same $C_{r}$ for some $\left[r_{0}, r_{1}\right]$, or 
equivalently if $q_{\lambda}$ and $q_{\lambda}^{\prime}$ both are on the dual curve of $C_{r}$. In fact the pencil of dual conics of $C_{r}$ is exactly the pencil of conics given by $\left\{r_{0}\left(L_{1}+L_{2}\right)+r_{1} B \mid\left[r_{0}, r_{1}\right] \in \mathbb{P}^{1}\right\}$. Summing up the arguments above, we obtain the following theorems.

THEOREM 3.1. Every rational elliptic surface that has a four torsion section can be obtained by the above method by choosing a suitable sub-pencil $\lambda$.

TheOREM 3.2. Any two surfaces $\mathcal{E}_{\lambda}$ and $\mathcal{E}_{\lambda^{\prime}}$ obtained in Lemma 3.3 are isomorphic as elliptic surfaces if and only if $q_{\lambda}$ and $q_{\lambda}^{\prime}$ lie on the same member of $\left\{r_{0}\left(L_{1}+L_{2}\right)+r_{1} B \mid\right.$ $\left.\left[r_{0}, r_{1}\right] \in \mathbb{P}^{1}\right\}$.

We note that since $\lambda$ is a $D_{8}$-invariant pencil, the $D_{8}$-action on $\mathbb{P}^{1} \times \mathbb{P}^{1}$ lifts to a relative $D_{8}$-action on $\mathcal{E}_{\lambda}$. We will investigate this action on $\mathcal{E}_{\lambda}$ in Section 5 .

\section{The height-pairing and Mordell-Weil lattices of elliptic surfaces}

In this section we state some facts on the height-pairing and Mordell-Weil lattices of elliptic surfaces which enable us to compute the intersection numbers of the sections of an elliptic surface. For details we refer the reader to [8], [10] and [11]. Recall that we assume that an elliptic surface has a section and at least one singular fiber.

Let $f: \mathcal{E} \rightarrow C$ be an elliptic surface. Let $F_{v}$ denote the fiber of $f$ over $v \in C$. Let

$$
\operatorname{Sing}(f)=\left\{v \in C \mid F_{v} \text { is singular }\right\}
$$

and

$$
R=\operatorname{Red}(f)=\left\{v \in C \mid F_{v} \text { is reducible }\right\} .
$$

We label each $F_{v}(v \in \operatorname{Red}(f))$ in the following way:

$$
F_{v}=\Theta_{v, 0}+\sum_{i=1}^{m_{v}-1} \mu_{v, i} \Theta_{v, i},
$$

where $\Theta_{v, i}$ are the irreducible components of $F_{v}, \mu_{v, i}$ is the multiplicity of $\Theta_{v, i}, m_{v}$ is the number of components and $\Theta_{v, 0}$ is the unique component intersecting the zero section.

For each $v \in R$, let $A_{v}$ be the intersection matrix of the components. Let $\operatorname{NS}(\mathcal{E})$ be the Néron-Severi group of $\mathcal{E}$. For each $s \in \operatorname{MW}(\mathcal{E})$ we denote both the corresponding curve on $\mathcal{E}$ and the divisor class in $\mathrm{NS}(\mathcal{E})$ by $[s]$. Note that $\left[s+s^{\prime}\right]$ is not equal to $[s]+\left[s^{\prime}\right]$ in general. We denote the intersection number of two divisors $D, D^{\prime}$ by $D . D^{\prime}$. We denote the arithmetic genus of $\mathcal{E}$ by $\chi$. Let $T$ be the subgroup of $\mathrm{NS}(\mathcal{E})$ generated by the classes of the zero section $[O]$ the fibers $F$ and $\Theta_{v, j}\left(v \in R, 1 \leq i \leq m_{v}-1\right)$ :

$$
\begin{aligned}
A_{v} & =\left(\left(\Theta_{v, i} \cdot \Theta_{v, j}\right)\right)_{1 \leq i, j \leq m_{v}-1}, \\
T & =\langle(O), F\rangle+\bigoplus_{v \in R}\left(\oplus_{i=1}^{m_{v}-1} \Theta_{v, i}\right) .
\end{aligned}
$$

Lemma 4.1. [11, Lemma 8.1] For any $s \in \mathrm{MW}(\mathcal{E})$, there exists a unique element of $\operatorname{NS}(\mathcal{E})_{\mathbb{Q}}$, say $\varphi(s)$, satisfying the conditions:

(1) $\varphi(s)=[s] \bmod T_{\mathbb{Q}}$; and

(2) $\varphi(s) \perp T$. 
Explicitly, $\varphi(s)$ is the class of the divisor $D_{s}$ with $\mathbb{Q}$-coefficients given by

$$
\begin{aligned}
D_{s}= & {[s]-[O]-([s] .[O]+\chi) F } \\
& +\sum_{v \in R}\left(\Theta_{v, 1}, \ldots, \Theta_{v, m_{v}-1}\right)\left(-A_{v}^{-1}\right)\left(\begin{array}{c}
{[s] \cdot \Theta_{v, 1}} \\
\vdots \\
{[s] . \Theta_{v, m_{v}-1}}
\end{array}\right) .
\end{aligned}
$$

Lemma 4.2. [11, Lemma 8.2] The map

$$
\varphi: \operatorname{MW}(\mathcal{E}) \rightarrow \mathrm{NS}(\mathcal{E})_{\mathbb{Q}}
$$

is a group homomorphism such that

$$
\operatorname{Ker}(\varphi)=\operatorname{MW}(\mathcal{E})_{\text {tor }}
$$

Lemma 4.3. [11, Theorem 8.4] For any $s, s^{\prime} \in \operatorname{MW}(\mathcal{E})$, let

$$
\left\langle s, s^{\prime}\right\rangle=-\varphi(s) \cdot \varphi\left(s^{\prime}\right) .
$$

Then it defines a symmetric bilinear pairing on $\mathrm{MW}(\mathcal{E})$, which induces the structure of a positive-definite lattice on $\mathrm{MW}(\mathcal{E}) / \mathrm{MW}(\mathcal{E})_{\text {tor. }}$.

Definition 4.1. The pairing in Lemma 4.3 is called the height-pairing and the pair

$$
\left(\mathrm{MW}(\mathcal{E}) / \mathrm{MW}(\mathcal{E})_{\mathrm{tor}},\langle,\rangle\right)
$$

is called the Mordell-Weil lattice of the elliptic surface $f: \mathcal{E} \rightarrow C$.

Note that we abuse notation and let $\langle s, t\rangle$ denote both the height-pairing of $s, t$ and a group generated by $s$ and $t$. It should be apparent from the context in which meaning $\langle s, t\rangle$ is used.

The height-pairing can be calculated explicitly by the following formula.

TheOrem 4.1. [11, Theorem 8.6] For any $s, s^{\prime} \in \operatorname{MW}(\mathcal{E})$,

$$
\begin{aligned}
& \left\langle s, s^{\prime}\right\rangle=\chi+[s] .[O]+\left[s^{\prime}\right] .[O]-[s] .\left[s^{\prime}\right]-\sum_{v \in R} \operatorname{contr}_{v}\left(s, s^{\prime}\right), \\
& \langle s, s\rangle=2 \chi+2[s] .[O]-\sum_{v \in R} \operatorname{contr}_{v}(s),
\end{aligned}
$$

where $\chi$ is the arithmetic genus of $\mathcal{E},[s] .\left[s^{\prime}\right]$ is the intersection number of sections $s$ and $s^{\prime}$, and $\operatorname{contr}_{v}\left(s, s^{\prime}\right)$ is the local contribution of reducible singular fibers given by

$$
\operatorname{contr}_{v}\left(s, s^{\prime}\right)= \begin{cases}\left(-A_{v}^{-1}\right)_{i, j} & \text { if } i \geq 1, j \geq 1, \\ 0 & \text { otherwise. }\end{cases}
$$

In the second formula, $\operatorname{contr}_{v}(s)=\operatorname{contr}_{v}(s, s)$.

The explicit values of the contribution term $\operatorname{contr}_{v}\left(s, s^{\prime}\right)$ are given in Table 4 from [11]. The second row gives values for $\operatorname{both}_{\operatorname{contr}}(s)$ and $\operatorname{contr}_{v}\left(s, s^{\prime}\right)$ for $i=j$.

The structure of the Mordell-Weil lattice of rational elliptic surfaces have been determined by Oguiso and Shioda [8]. Table 5 is an excerpt from the list given in [8] of surfaces that have a $n$-torsion section $(n \geq 4)$.

In the table, $A_{1}^{*}$ denotes the dual lattice of the root lattice of type $A_{1}$. In explicit terms, $A_{1}^{*}$ is a lattice of rank one generated by an element $s$ such that $\langle s, s\rangle=1 / 2$. 
TABLE 4. The values of the contribution term.

\begin{tabular}{|c|c|c|c|c|c|c|c|}
\hline Type of $F_{v}$ & $I I I$ & $I I I^{*}$ & $I V$ & $I V^{*}$ & $I_{b}(b \geq 2)$ & \multicolumn{2}{|c|}{$I_{b}^{*}(b \geq 0)$} \\
\hline $\begin{array}{c}\operatorname{contr}_{v}(s) \\
\operatorname{contr}_{v}\left(s, s^{\prime}\right)\end{array}$ & $1 / 2$ & $3 / 2$ & $2 / 3$ & $4 / 3$ & $i(b-i) / b$ & $\left\{\begin{array}{l}1 \\
1+b / 4\end{array}\right.$ & $\begin{array}{l}(i=1) \\
(i>1)\end{array}$ \\
\hline$(i<j)$ & - & - & $1 / 3$ & $2 / 3$ & $i(b-j) / b$ & $\left\{\begin{array}{l}1 / 2 \\
(2+b) / 4\end{array}\right.$ & $\begin{array}{l}(i=1) \\
(i>1)\end{array}$ \\
\hline
\end{tabular}

TABLE 5. The structure of the Mordell-Weil lattice.

\begin{tabular}{cccc}
\hline No. & Singular fibers & $\mathrm{MW}(\mathcal{E})$ & Mordell-Weil lattice \\
\hline 66 & $I_{6}, I_{3}, I_{2}, I_{1}$ & $\mathbb{Z} / 6 \mathbb{Z}$ & 0 \\
67 & $I_{5}, I_{5}, I_{1}, I_{1}$ & $\mathbb{Z} / 5 \mathbb{Z}$ & 0 \\
70 & $I_{8}, I_{2}, I_{1}, I_{1}$ & $\mathbb{Z} / 4 \mathbb{Z}$ & 0 \\
72 & $I_{1}^{*}, I_{4}, I_{1}$ & $\mathbb{Z} / 4 \mathbb{Z}$ & 0 \\
74 & $I_{4}, I_{4}, I_{2}, I_{2}$ & $\mathbb{Z} / 2 \mathbb{Z} \oplus \mathbb{Z} / 4 \mathbb{Z}$ & 0 \\
58 & $I_{4}, I_{4}, I_{2}, I_{1}, I_{1}$ & $\mathbb{Z} \oplus \mathbb{Z} / 4 \mathbb{Z}$ & $A_{1}^{*}$ \\
\hline
\end{tabular}

Lemma 4.4. [6, Proposition VII.3.2] Let $t$ and $t^{\prime}$ be two torsion sections of a rational elliptic surface. Then $[t] .\left[t^{\prime}\right]=0$.

\section{Birational classification of rational elliptic surfaces}

The classification of (minimal) rational $G$-surfaces up to birational equivalence has been done by Dolgachev and Iskovskikh in [2]. They find all minimal $G$-surfaces and use the classification of $G$-equivariant elementary links to determine whether two minimal $G$ surfaces are equivalent or not.

On the other hand many interesting $G$-surfaces, including elliptic surfaces, have a nonminimal $G$-action. Although their birational classification is essentially done in [2], it is not easy to point out where non-minimal surfaces fit in the classification. In this section, we clarify where rational elliptic $D_{8}$-surfaces fit in Dolgachev and Iskovskik's classification, determine their birational equivalence classes and prove Theorems 1.1 and 1.2. First we find the corresponding minimal $D_{8}$-surfaces.

PROPOSITION 5.1. Let $\mathcal{E}$ be a rational elliptic surface of type 70,72 or 74 with relative $D_{8}$-action. Then $\mathcal{E}$ can be $D_{8}$-equivariantly blown-down to $\mathbb{P}^{1} \times \mathbb{P}^{1}$.

Proof. If $\mathcal{E}$ is of type 74, there are eight torsion sections. By Lemma 4.4 the torsion sections of a rational elliptic surface are mutually disjoint, hence we can blow-down these eight sections. Similarly, if $\mathcal{E}$ is of type 72 we can blow-down the torsion sections and then blow-down the simple components of the singular fiber of type $I_{1}^{*}$. If $\mathcal{E}$ is of type 74 we can blow-down the torsion sections and then the components of the singular fiber of type $I_{8}$ that intersect with the torsion sections. Since we are contracting $D_{8}$-orbits, the blow-down morphism is $D_{8}$-equivariant. 
In each case the resulting surface is isomorphic to $\mathbb{P}^{1} \times \mathbb{P}^{1}$ since the degree is 8 and the image of a singular fiber of type $I_{4}$ or $I_{8}$ becomes a cycle of four non-singular rational curves whose components have self-intersection number 0 .

To determine the $D_{8}$-minimal models of surfaces of type 58 , we need to study how the sections intersect each other in detail. We use the following lemmas. Let $G\left(F_{v}\right)$ be the finite group corresponding to the singular fiber $F_{v}$. See $[4,10,11]$ for details.

Lemma 5.1. [10, Lemma 7.4] The map $\psi: E(K) \rightarrow \prod G\left(F_{v}\right)$ that sends sections to the simple components which the sections intersect, is a group homomorphism.

LEMMA 5.2. [10, Corollary 7.5] The restriction of $\psi$ to torsion points is an injection.

Let $\mathcal{E}$ be an elliptic surface of type 58. The singular fibers of $\mathcal{E}$ are $I_{4}, I_{4}, I_{2}, I_{1}, I_{1}$. Hence $\prod G\left(F_{v}\right)=(\mathbb{Z} / 4 \mathbb{Z})^{\oplus 2} \oplus \mathbb{Z} / 2 \mathbb{Z}$. Let $t$ be a four-torsion section. Then $\langle t, t\rangle=0$ and $[t] .[O]=0$ since $t$ is a torsion element and by Lemma 4.4. On the other hand

$$
\langle t, t\rangle=2+2[t] .[O]-\sum_{v} \operatorname{contr}_{v}(t)
$$

by Theorem 4.1. Hence we have $\sum_{v} \operatorname{contr}_{v}(t)=2$. By looking at the table of contribution terms (Table 4), there are two possibilities for $\psi(t)$ (after renumbering the components if necessary), namely $\psi(t)=(2,2,0)$ or $(1,1,1)$, but the first possibility is ruled out because $t$ is a four-torsion section and $\psi$ is injective when restricted to the group of torsion sections. Now we can suppose that $\psi(t)=(1,1,1)$. The Mordell-Weil lattice of $\mathcal{E}$ is isomorphic to $A_{1}^{*}$. Let $s$ be a section corresponding to the generator of $A_{1}^{*}$. Then $\langle s, s\rangle=1 / 2$. Again by the formula for the height-pairing we have

$$
\frac{1}{2}=\langle s, s\rangle=2+2[s] .[O]-\sum_{v} \operatorname{contr}_{v}(s) .
$$

Since $\sum_{v} \operatorname{contr}_{v}(s) \leq 5 / 2$ we have $[s]$. [O] $=0$ and $\sum_{v} \operatorname{contr}_{v}(s)=3 / 2$. Similarly for $\langle s, t\rangle$, we find that $[s] .[t]=0$ and $\sum_{v} \operatorname{contr}_{v}(s, t)=1$. We have four possibilities for $\psi(s)$, namely $(3,1,0),(0,2,1),(1,3,0),(2,0,1)$ but they are equivalent modulo $\psi(t)$ so we can assume that $\psi(s)=(3,1,0)$. The following lemmas can be proved by using the formula for the height-pairing in a similar way.

LEMMA 5.3. The sections in the set $\{[O],[t],[2 t],[3 t],[s],[s+t],[s+2 t],[s+3 t]\}$ are mutually disjoint.

LEMmA 5.4. The sections in the set $\{[ \pm k s],[ \pm k s+t],[ \pm k s+2 t],[ \pm k s+3 t]\}$ are not mutually disjoint for any $k>0$.

PROPOSITION 5.2. Let $\mathcal{E}$ be a rational elliptic surface of type 58.

(1) A D8-action of type 1 on $\mathcal{E}$ cannot be equivariantly blown-down to $\mathbb{P}^{1} \times \mathbb{P}^{1}$. Furthermore, the $D_{8}$-surface $\Sigma$ obtained by equivariantly blowing-down $\operatorname{Orb}_{D_{8}}([O])$ $=\{[O],[t],[2 t], 3 t]\}$ is a Del Pezzo surface of degree 4 with minimal $D_{8}$-action.

(2) A $D_{8}$-action of type 2 on $\mathcal{E}$ can be equivariantly blown-down to $\mathbb{P}^{1} \times \mathbb{P}^{1}$.

Proof. In the first case, we can equivariantly blow-down the $D_{8}$-orbit $\operatorname{Orb}_{D_{8}}([O])=$ $\{[O],[t],[2 t],[3 t]\}$ of $[O]$ because it is mutually disjoint by Lemma 5.3. Let $K_{\mathcal{E}}$ 
(respectively $K_{\Sigma}$ ) be the canonical divisor of $\mathcal{E}$ (respectively $\Sigma$ ). By the canonical bundle formula for elliptic surfaces we have $-K_{\mathcal{E}} \sim F$, where $F$ is the class of a fiber. Hence $-K_{\mathcal{E}} . C \geq 0$ for all irreducible curves $C$. Let $v: \mathcal{E} \rightarrow \Sigma$ be the blow-down morphism. Then

$$
K_{\mathcal{E}}=v^{*} K_{\Sigma}+[O]+[t]+[2 t]+[3 t] .
$$

Suppose there exists an irreducible curve $C^{\prime}$ on $\Sigma$ such that $-K_{\Sigma} \cdot C^{\prime} \leq 0$. Let $v^{*} C^{\prime}=$ $\tilde{C}^{\prime}+c_{1}[O]+c_{2}[t]+c_{3}[2 t]+c_{4}[3 t]$, where $\tilde{C}^{\prime}$ is the proper transform of $C$ under $v$ and $c_{i} \geq 0$. Then

$$
0 \leq-K_{\mathcal{E}} \cdot \tilde{C}^{\prime}=-K_{\Sigma} \cdot C^{\prime}-\left(c_{1}+c_{2}+c_{3}+c_{4}\right) .
$$

Hence we have $c_{1}=\cdots=c_{4}=-K_{\mathcal{E}} \cdot \tilde{C}^{\prime}=0$ which implies that $\tilde{C}^{\prime}$ is a fiber component that does not meet $[O],[t],[2 t],[3 t]$, but this is impossible. Hence $-K_{\Sigma} \cdot C^{\prime}>0$ for all irreducible curves $C^{\prime}$ and $-K_{\Sigma}$ is ample by Nakai's criterion for ampleness [3].

It is well known that there are 16 exceptional curves on a Del Pezzo surface of degree 4. In our case, they are the images of the curves $\{[ \pm s],[ \pm s+t],[ \pm s+2 t],[ \pm s+3 t]\}$ and the images of the components of singular fibers of type $I_{4}$. By Lemma 5.4 , there are no $D_{8}$-orbits consisting of disjoint $(-1)$-curves, hence $\mathcal{E}^{\prime}$ is a minimal $D_{8}$-surface.

In the second case, $\operatorname{Orb}_{D_{8}}([O])=\{[O],[t],[2 t],[3 t],[s],[s+t],[s+2 t],[s+3 t]\}$ and these sections are mutually disjoint by Lemma 5.3 . Hence we can equivariantly blowdown $\operatorname{Orb}_{D_{8}}([O])$. The resulting surface is isomorphic to $\mathbb{P}^{1} \times \mathbb{P}^{1}$ since it is of degree 8 and the image of a singular fiber of type $I_{4}$ becomes a cycle of rational curves whose components have self-intersection number 0 .

Propositions 5.1 and 5.2 tell us about the underlying surface of the $D_{8}$-minimal models of $\mathcal{E}$, but they do not tell us about the $D_{8}$-action on the surface. We use the explicit description of $\mathcal{E}$ as a blow-up of $\mathbb{P}^{1} \times \mathbb{P}^{1}$ from Section 3 to determine the group action on the $D_{8}$-minimal models.

PRoposition 5.3. Let $\mathcal{E}$ be a rational elliptic $D_{8}$-surface of type 70 or 72 with the unique $D_{8}$-action, or, of type 58 or 74 with $D_{8}$-action of type 2 . Then $\mathcal{E}$ is birationally equivalent as a $D_{8}$-surface to $\mathbb{P}^{1} \times \mathbb{P}^{1}$ with the following $D_{8}=\langle\sigma, \tau\rangle$ action:

$$
\begin{aligned}
& \sigma\left(\left[y_{0}, y_{1}\right],\left[z_{0}, z_{1}\right]\right)=\left(\left[z_{0}, z_{1}\right],\left[y_{0}, y_{1}\right]\right), \\
& \tau\left(\left[y_{0}, y_{1}\right],\left[z_{0}, z_{1}\right]\right)=\left(\left[z_{0}, z_{1}\right],\left[y_{1}, y_{0}\right]\right) .
\end{aligned}
$$

Proof. By Theorem 3.1 we know that every underlying $\mathcal{E}$ can be obtained as a blow-up of $\mathbb{P}^{1} \times \mathbb{P}^{1}$. We need to check whether the relative $D_{8}$-action on $\mathcal{E}$ that is induced by the $D_{8}$-action on $\mathbb{P}^{1} \times \mathbb{P}^{1}$ coincides with the desired action.

If $\mathcal{E}$ is of type 70 or 72 there is nothing to prove since then $\mathcal{E}$ has a unique relative $D_{8}$-action. If $\mathcal{E}$ is of type 74 we see that the induced action is of type 2 because the $D_{8}$-action on $\mathbb{P}^{1} \times \mathbb{P}^{1}$ acts transitively on the base points of the pencil corresponding to $\mathcal{E}$ and hence the induced action on $\mathcal{E}$ must be transitive on $\operatorname{MW}(\mathcal{E})$. If $\mathcal{E}$ is of type 58 then we see that the action must be of type 2 because actions of type 1 cannot be equivariantly blown-down to $\mathbb{P}^{1} \times \mathbb{P}^{1}$ by Proposition 5.2. 
PROpOSITION 5.4. Let $\mathcal{E}$ be a rational elliptic $D_{8}$-surface of type 74 with action of type 1. Then $\mathcal{E}$ is birationally equivalent to $\mathbb{P}^{1} \times \mathbb{P}^{1}$ with the following $D_{8}=\left\langle\sigma^{\prime}, \tau^{\prime}\right\rangle$ action:

$$
\begin{aligned}
\sigma^{\prime}\left(\left[y_{0}, y_{1}\right],\left[z_{0}, z_{1}\right]\right) & =\left(\left[z_{0}-\sqrt{-1} z_{1}, \sqrt{-1} z_{0}-z_{1}\right],\left[y_{0}-\sqrt{-1} y_{1}, \sqrt{-1} y_{0}-y_{1}\right]\right), \\
\tau^{\prime}\left(\left[y_{0}, y_{1}\right],\left[z_{0}, z_{1}\right]\right) & =\left(\left[z_{0}, z_{1}\right],\left[y_{1}, y_{0}\right]\right) .
\end{aligned}
$$

Proof. Let $C_{0}$ and $C_{1}$ be defined by

$$
\begin{aligned}
& C_{0}: 4 y_{0} y_{1} z_{0} z_{1}-y_{0}^{2} z_{1}^{2}-y_{0}^{2} z_{0}^{2}-y_{1}^{2} z_{0}^{2}-y_{1}^{2} z_{1}^{2}=0, \\
& C_{1}: y_{0} y_{1} z_{0}^{2}+y_{0} y_{1} z_{1}^{2}+z_{0} z_{1} y_{0}^{2}+z_{0} z_{1} y_{1}^{2}=0 .
\end{aligned}
$$

Then the pencil $\lambda=\left\{\lambda_{0} C_{0}+\lambda_{1} C_{1}\right\}$ is a sub-pencil of $\Lambda$ which was defined in Section 3. It can be easily checked that $\lambda$ is invariant under the above $D_{8}$-action, the elliptic surface $\mathcal{E}_{\lambda}$ obtained by blowing-up the base points is of type 74 , and that the $D_{8}$-action on $\mathbb{P}^{1} \times \mathbb{P}^{1}$ is not transitive on the base points of $\lambda$, hence the induced relative $D_{8}$-action on $\mathcal{E}_{\lambda}$ is not transitive on $\operatorname{MW}(\mathcal{E})$. Hence it must be of type 1 .

Now that we have found the corresponding minimal models of each $\mathcal{E}$, we can see where rational elliptic surfaces fit in Dolgachev and Iskovskikh's classification. According to the classification of elementary links given in [2], the two $D_{8}$-actions on $\mathbb{P}^{1} \times \mathbb{P}^{1}$ given in Propositions 5.3 and 5.4 are birationally distinct. We look into the case of surfaces of type 58 with $D_{8}$-action of type 1 in detail.

Let $\mathcal{E}$ be a rational elliptic surface of type 58 with $D_{8}$-action of type 1 . Then we know from Section 3 that it can be presented as a blow-up of $\mathbb{P}^{1} \times \mathbb{P}^{1}$ at eight points. Let $v: \mathcal{E} \rightarrow \mathbb{P}^{1} \times \mathbb{P}^{1}$ be the blow-up morphism. We label the eight points by

$$
\begin{gathered}
p_{1}=([a, b],[c, d]), \quad p_{2}=\tau\left(p_{1}\right), \quad p_{3}=\tau^{2}\left(p_{1}\right), \quad p_{4}=\tau^{3}\left(p_{1}\right), \\
p_{5}=\sigma\left(p_{1}\right), \quad p_{6}=\tau \sigma\left(p_{1}\right), \quad p_{7}=\tau^{2} \sigma\left(p_{1}\right), \quad p_{8}=\tau^{3} \sigma\left(p_{1}\right),
\end{gathered}
$$

where $\sigma, \tau$ are defined as in Section 3. We can suppose that $v([s])=p_{1}, v([s+t])=p_{2}$, $v([s+2 t])=p_{3}, v([s+3])=p_{4}, v([o])=p_{5}, v([t])=p_{6}, v([2 t])=p_{7}$ and $v([3 t])=p_{8}$. Let $\Sigma$ be the $D_{8}$-minimal Del Pezzo surface of degree 4 corresponding to $\mathcal{E}$. Then $\Sigma$ is the blow-up of $\mathbb{P}^{1} \times \mathbb{P}^{1}$ centered at $p_{1}, p_{2}, p_{3}, p_{4}$. The inverse images of $([1,1],[1,1])$ and $([1,-1],[1,-1])$ give $D_{8}$-fixed points which correspond to the singular points of the singular fibers of type $I_{1}$. Let $\Sigma^{\prime}$ be the blow-up of $\Sigma$ centered at the inverse image of $([1,1],[1,1])$. The proper transform of a singular fiber of type $I_{1}$ on $\Sigma^{\prime}$ is a $D_{8}$-invariant smooth rational curve with self-intersection number 0 . Hence there exists a $D_{8}$-invariant conic bundle structure on $\Sigma^{\prime}$. Let $v^{\prime}: \Sigma^{\prime} \rightarrow \mathbb{P}^{1} \times \mathbb{P}^{1}$ be the blow-up centered at $p_{1}, p_{2}, p_{3}, p_{4}, q=([1,1],[1,1])$. Then the conic bundle structure on $\Sigma^{\prime}$ is induced by the proper transform of curves of bi-degree $(2,2)$ on $\mathbb{P}^{1} \times \mathbb{P}^{1}$ passing through $p_{1}, p_{2}, p_{3}, p_{4}$ with multiplicity one and $q$ with multiplicity two.

Let $C_{\mathrm{ij}}$ be the curve of bi-degree $(1,1)$ in $\mathbb{P}^{1} \times \mathbb{P}^{1}$ passing through $p_{i}, p_{j}$ and ([1, 1], $[1,1]$ ). Let $C_{1}$ (respectively $C_{2}$ ) be the unique curve of bi-degree $(2,1)$ (respectively $(1,2))$ passing through $p_{1}, p_{2}, p_{3}, p_{4}, q$. Let $L_{1}$ be $z_{0}-z_{1}=0$ and $L_{2}$ be $y_{0}-y_{1}=0$. Then the singular fibers of the conic bundle on $\Sigma^{\prime}$ are given by the proper transforms of $C_{1}+L_{1}, C_{2}+L_{2}, C_{12}+C_{34}, C_{13}+C_{24}$ and $C_{14}+C_{23}$. By looking at the intersection 
with the curves $\{[ \pm s],[ \pm s+t],[ \pm s+2 t],[ \pm s+3 t]\}$ one finds that $\iota$ and $t$ act on the set $\left\{C_{i}, L_{j}, C_{k l}\right\}$ as follows:

$$
\begin{aligned}
& \iota\left(C_{1}\right)=L_{2}, \quad \iota\left(C_{2}\right)=L_{1}, \quad \iota\left(C_{12}\right)=C_{23}, \quad \iota\left(C_{34}\right)=C_{14}, \quad \iota\left(C_{13}\right)=C_{24}, \\
& t\left(C_{1}\right)=C_{2}, \quad t\left(C_{2}\right)=C_{1}, \quad t\left(L_{1}\right)=L_{2}, \quad t\left(L_{2}\right)=L_{1}, \\
& t\left(C_{12}\right)=C_{23}, \quad t\left(C_{23}\right)=C_{34}, \quad t\left(C_{34}\right)=C_{13}, \quad t\left(C_{14}\right)=C_{12}, \\
& t\left(C_{13}\right)=C_{24}, \quad t\left(C_{24}\right)=C_{13} .
\end{aligned}
$$

Lemma 5.5. Let $\mathcal{E}$ be a rational elliptic $D_{8}$-surface of type 58 with $D_{8}$-action of type 1 obtained by blowing-up $\mathbb{P}^{1} \times \mathbb{P}^{1}$ at $([a, b],[c, d])=p_{1}, p_{2}, \ldots, p_{8}$. Then the involutions $\iota \circ t$ and $\iota \circ t^{3}$ of $\mathcal{E}$ respectively fix a curve $C, C^{\prime}$ of genus 1 with the same $j$-invariant given by

$$
j(C)=j\left(C^{\prime}\right)=\frac{256 n_{1}^{3} n_{2}^{3}}{d_{1}^{4} d_{2}^{4} d_{3}^{2}}
$$

where

$$
\begin{aligned}
& n_{1}=a^{2} c^{2}+a^{2} d^{2}+b^{2} c^{2}+b^{2} d^{2}+a^{2} c d+b^{2} c d-a b c^{2}-a b d^{2}-4 a b c d \\
& n_{2}=a^{2} c^{2}+a^{2} d^{2}+b^{2} c^{2}+b^{2} d^{2}-a^{2} c d-b^{2} c d+a b c^{2}+a b d^{2}-4 a b c d \\
& d_{1}=a c-b d \\
& d_{2}=a d-b c \\
& d_{3}=a^{2} d^{2}+a^{2} c^{2}+b^{2} c^{2}+b^{2} d^{2}-4 a b c d .
\end{aligned}
$$

Proof. Let $F_{1}=C_{1}+L_{1}, F_{2}=C_{2}+L_{2}, F_{3}=C_{12}+C_{34}, F_{4}=C_{13}+C_{24}$ and $F_{5}=C_{14}+$ $C_{23}$ be the five singular fibers of the conic bundle structure on $\mu: \Sigma^{\prime} \rightarrow \mathbb{P}^{1}$.

By using Maple we can show that it is possible to choose coordinates so that $\mu\left(F_{1}\right)=$ $[1,0], \mu\left(F_{2}\right)=[0,1] \mu\left(F_{3}\right)=\left[(a c-d b)^{2},(a d-b c)^{2}\right], F_{4}=[1,-1]$ and $F_{5}=[(a d-$ $\left.b c)^{2},(a c-d b)^{2}\right]$.

Since $\iota \circ t$ fixes two points on a general fiber and since it interchanges the components of $F_{1}, F_{2}, F_{3}$, and $F_{4}$, the restriction of the structure map to the fixed curve $C$ is a double cover branched at $[1,0],[1,-1],[0,1]$ and $\left[(a c-d b)^{2},(a d-b c)^{2}\right]$. Hence $C$ is of genus 1 with the desired $j$-invariant. The case of $\iota \circ t^{3}$ can be calculated in a similar way.

The two involutions $\iota \circ t$ and $\iota \circ t^{3}$ are cubic involutions in Blanc's terminology [1]. Since the fixed curves of $\iota \circ t$ and $\iota \circ t^{3}$ are of positive genus the $D_{8}$-minimal Del Pezzo surfaces of degree 4 obtained above are birationally distinct to the two $D_{8}$-actions on $\mathbb{P}^{1} \times \mathbb{P}^{1}$ given in Propositions 5.3 and 5.4 because the latter two have only rational curves as fixed curves.

PRoposition 5.5. Let $\mathcal{E}$, $\mathcal{E}^{\prime}$ be rational elliptic $D_{8}$-surfaces of type 58 with $D_{8}$-action of type 1 . Then $\mathcal{E}$ and $\mathcal{E}^{\prime}$ are birationally equivalent as $D_{8}$-surfaces if and only if $\mathcal{E}$ and $\mathcal{E}^{\prime}$ are isomorphic as elliptic surfaces.

Proof. This is due to [1, Proposition 8.1.8], which states that two Del Pezzo surfaces of degree 4 with $(\mathbb{Z} / 2 \mathbb{Z})^{2}$-action generated by two cubic involutions are birationally equivalent as $(\mathbb{Z} / 2 \mathbb{Z})^{2}$-surfaces if and only if there exists a $(\mathbb{Z} / 2 \mathbb{Z})^{2}$-equivariant isomorphism between them. In our case, if $\mathcal{E}, \mathcal{E}^{\prime}$ are birationally equivalent as $D_{8}$-surfaces, the Del Pezzo surfaces of 
degree 4 corresponding to them become isomorphic as $D_{8}$-surfaces by [1, Proposition 8.1.8] and hence $\mathcal{E}, \mathcal{E}^{\prime}$ are isomorphic as $D_{8}$-surfaces.

We note that two non-isomorphic surfaces $\mathcal{E}$ and $\mathcal{E}^{\prime}$ may have fixed curves with the same $j$-invariant. This is another example of non-birationally equivalent surfaces that have the same set of fixed points.

The arguments in this section add up to the proof of Theorem 1.1. Theorem 1.2 is a direct consequence of Theorems 1.1 and 3.2.

\title{
REFERENCES
}

[1] J. Blanc. Finite abelian subgroups of the Cremona group of the plane. Thesis, University of Geneva, 2006. Available online at http://www.unige.ch/cyberdocuments/theses2006/BlancJ/meta.htm.

[2] I. V. Dolgachev and V. A. Iskovskikh. Finite subgroups of the plane Cremona group. In Algebra, Arithmetic and Geometry: in Honor of Yu. I. Manin, Vol. I (Progress in Mathematics, 269). Birkhäuser, Boston, 2009, pp. 443-548.

[3] R. Hartshorne. Algebraic Geometry (Graduate Texts in Mathematics, 52). Springer, 1977.

[4] K. Kodaira. On compact analytic surfaces II. Ann. of Math. 77 (1963), 563-626.

[5] K. Kodaira. On compact analytic surfaces III. Ann. of Math. 78 (1963), 1-40.

[6] R. Miranda. The basic theory of rational elliptic surfaces. Dottorato di Ricerca in Mathematica. Dipartmento di Mathematica dell'Universià di Pisa, 1989.

[7] R. Miranda and U. Persson. On extremal rational elliptic surfaces. Math. Z. 193 (1986), 537-558.

[8] K. Oguiso and T. Shioda. The Mordell-Weil lattice of a rational elliptic surface. Comment. Math. Univ. St. Pauli 40 (1991), 83-99.

[9] U. Persson. Configuration of singular fibers on rational elliptic surfaces. Math. Z. 205 (1990), 1-47.

[10] M. Schütt and T. Shioda. Elliptic surfaces. Algebraic Geometry in East Asia-Seoul 2008 (Adv. Stud. Pure Math., 60). Mathematical Society of Japan, Tokyo, 2010, 51-160.

[11] T. Shioda. On the Mordell-Weil lattices. Comment. Math. Univ. St. Pauli 39 (1990), 211-240.

[12] J. H. Silverman. The Arithmetic of Elliptic Curves (Graduate Texts in Mathematics, 106). Springer, 1986.

[13] J. H. Silverman. Advanced Topics in the Arithmetic of Elliptic Curves (Graduate Texts in Mathematics, 151). Springer, 1994.

[14] H. Tokunaga. A remark on Artal's paper. Kodai Math. J. 19 (1996), 207-217.

[15] H. Tokunaga. Dihedral coverings branched along maximizing sextics. Math. Ann. 308 (1997), 633-648.

[16] H. Tokunaga. Some examples of Zariski pairs arising from certain elliptic K3 surfaces. Math. Z. 227 (1998), 465-477; Some examples of Zariski pairs arising from certain elliptic K3 surfaces, II: Degtyarev's conjecture. Math. Z. 230 (1999), 389-400.

[17] H. Tokunaga. Dihedral covers and an elementary arithmetic on elliptic surfaces. J. Math. Kyoto Univ. 44 (2004), 255-270.

[18] T. Yasumura. Triple coverings over the projective plane whose branch loci are quintic curves. Thesis, Tokyo Metropolitan University, 2011.

\author{
Shinzo Bannai \\ Department of Mathematics and Information Sciences \\ Graduate School of Science and Engineering \\ Tokyo Metropolitan University \\ 1-1 Minami-Ohsawa \\ Hachiohji 192-0397 \\ Japan \\ (E-mail: shinzo.bannai@gmail.com)
}

